\title{
On the Ability to Inhibit Simple Thoughts and Actions: II. Stop-Signal Studies of Repetition Priming
}

\author{
Gordon D. Logan \\ Purdue University
}

\begin{abstract}
When a person inhibits a simple action that is based on a simple thought, does the thought stop with the action? Five experiments were conducted to address that question, attempting to determine the conditions under which simple thoughts could be inhibited. Subjects made speeded category and rhyme judgments about word pairs and were asked to inhibit their (overt) responses on $40 \%$ of the trials. The word pairs were presented again, and priming from repetition was used to decide whether the original thoughts went on to completion when their overt responses were inhibited. The results suggested that thoughts could be inhibited by switching to a new task when the response was inhibited (Experiment 2) or by changing the display when the response was inhibited (Experiment 4) but not by simply inhibiting the response (Experiment 1). The results are compared with other studies that used recognition memory as the index of inhibited thought, and implications for the basis of repetition priming are discussed.
\end{abstract}

Cognitive control depends to a large extent on the ability to inhibit courses of thought and action that are not relevant to current goals. Some thoughts and actions may never be relevant to current goals; others may have been relevant but are no longer so. This article reports an investigation of the inhibition of the latter type of thought and action. Subjects were shown pairs of words and were asked to make category and rhyme judgments about them (e.g., Is a FRIGATE a BOAT? Does SLEIGH rhyme with PLAY?), which they reported by pressing keys. Occasionally, they were given a stop signal (a tone) which told them to inhibit their keypress response on that trial. Previous studies showed that subjects can inhibit a variety of responses up to the point of executing them (see Logan \& Cowan, 1984, for a review). The major question addressed in the present in-

This research was supported by Grant No. U0053 from the Natural Sciences and Engineering Research Council of Canada. I would like to thank Jane Zbrodoff for helpful comments and suggestions and Ken Davis and Randy Tonks for running the subjects and helping with the analysis. I am grateful to Richard Shiffrin, Larry Jacoby, Mike Masson, Tim Fuestel, Jim Neely, and Henry Roediger for helpful comments on the article.

Requests for reprints should be sent to Gordon D. Logan, Department of Psychological Sciences, Purdue University, West Lafayette, Indiana 47907. vestigation was whether the thoughts underlying the category and rhyme judgments were inhibited with the overt actions.

In order to determine whether the underlying thoughts were inhibited with the overt actions, the thoughts had to be made observable in some way. In a previous article, I used memory for the words whose responses were inhibited as an observable index of thought (Logan, 1983). The idea was that inhibited thoughts should lay down less complete memory traces than completed thoughts, and so should be remembered less accurately. Thus, if thoughts were inhibited with the actions, memory for words whose responses were inhibited should be worse than memory for words whose responses were not inhibited. However, if the thoughts ran on to completion ballistically when the actions were inhibited, memory for words whose responses were inhibited should be just as good as memory for words whose responses were not inhibited. A test of recognition memory suggested, for the most part, that the simple thoughts underlying category and rhyme judgments went on to completion when the corresponding responses were inhibited.

The present investigation extends the previous one by using repetition priming as the index of thought rather than recognition memory. Repetition priming refers to a re- 
duction in the time required to make a judgment the second time it is made (cf. Scarborough, Cortese, \& Scarborough, 1977).' It is interesting because it may reflect different aspects of memory performance than recognition memory does. Some claim it taps a different memory system than recognition memory does (e.g., Tulving, Schacter, \& Stark, 1982), whereas others claim it taps different information in the same memory system (e.g., Jacoby, 1983). In either case, repetition priming may provide information about the inhibition of thought that could not be assessed in the previous studies of recognition memory, which justifies the present investigation.

The hypothesis that thoughts are inhibited with their corresponding actions was assessed in two ways: First, the amount of repetition priming observed for word pairs presented with a stop signal was compared with the amount observed for word pairs presented without a stop signal. If subjects inhibited the underlying thoughts when they inhibited the overt actions, there should be less repetition priming for the former, stop-signal word pairs than for the latter, no-signal word pairs. However, if the underlying thoughts ran on to completion ballistically whether or not the corresponding actions were inhibited, then word pairs presented with a stop signal should produce as much repetition priming as word pairs presented without a stop signal. Second, the repetition priming produced by word pairs presented with a stop signal was assessed as a function of stop-signal delay (i.e., the interval between the onset of the word pair and the onset of the stop signal). Subjects should be more likely to inhibit their responses the sooner the stop signal occurred (see Logan \& Cowan, 1984). If they inhibited the underlying thought with the overt action, then word pairs presented with stop signals at short delays should produce less repetition priming than word pairs presented with stop signals at long delays; the amount of repetition priming should increase as stop-signal delay increases. However, if the underlying thoughts run on to completion ballistically whether or not the corresponding actions are inhibited, then the same amount of repetition priming should be observed at each stop-signal delay; repetition priming should be independent of stop-signal delay.
Five experiments were conducted. On each trial of each experiment, subjects saw a pair of words, one above the other. In the category task, they decided whether the bottom word was a member of the category named by the top word (e.g., PROFESSION/PROFESSOR) and in the rhyme task, they decided whether the bottom word rhymed with the top word (e.g., SIGN/LINE). The trials were organized into orienting blocks and repetition blocks. Subjects began by performing an orienting block in which a new set of word pairs was presented, with stop signals occurring at various delays on $40 \%$ of the trials. Then they performed a repetition block in which some of the word pairs were repeated and some were new, and no stop signals were presented. Repetition priming was assessed in the repetition block by subtracting reaction times to repeated word pairs from reaction times to new word pairs. Subjects then performed another orienting block and another repetition block with the same task (i.e., category or rhyme judgment) and then performed two more orienting and repetition blocks with the other task (i.e., rhyme or category judgment).

In Experiment 1, subjects were told simply to stop their overt response to the word pair when the stop signal occurred. In Experiment 2 , they were told to inhibit their response to the word pair and to make a different overt response to the stop signal when it occurred. Experiment 3 was conducted as a control for Experiment 2; subjects were told to respond overtly to the stop signal when it occurred but they were not told to inhibit their response to the word pair. In Experiment 4, subjects were told to inhibit their response to the word pair when the stop signal sounded but the word pair was extinguished and replaced by two strings of $X$ s when the stop signal sounded. Experiment 5 was a control for Experiment 4; the

\footnotetext{
1 Repetition priming may be defined more generally as the effect of a prior presentation of subsequent processing. Thus, it may be observed with dependent variables other than reaction time. For example, Jacoby and Dallas (1981) found that a prior presentation of a word increased the probability that it would be identified in a brief tachistoscopic exposure, and Tulving, Schacter, and Stark (1982) found that a prior presentation of a word increased the probability that it would be used to solve a word-fragment problem (e.g., G-N-O-A = gondola).
} 
word pair was extinguished and replaced with $X$ s when the stop signal sounded but subjects were not told to inhibit their responses to the word pair. The five experiments used the same basic procedure, so they will be described in a general method section.

\section{General Method}

\section{Subjects}

A separate group of 32 subjects served in each experiment. Most of the subjects were students from Introductory Psychology who served for course credit. A few subjects in each experiment came from the general university population and were paid $\$ 4$ for serving in the experiment. All subjects were naive as to the purpose of the experiment.

\section{Apparatus and Stimuli}

The stimuli were two sets of 280 words divided into 14 categories containing 20 members each. The words for the category task were selected from the Battig and Montague (1969) norms, excluding the five most frequent members of the categories, words that were longer than 12 letters, words that could be members of more than one category (e.g., palm), and category members that were more than one word long (e.g., spanish onion). The 280 words chosen ranged in length from 3 to 11 letters, averaging 6.29. The category names were fish, bird, profession, mammal, sport, vegetable, (musical) instrument, clothing, body part, weather, tree, boat, fruit, and (cooking) utensil. Each word was paired with its category name for positive (i.e., yes) category judgments and with the name of a different category for negative (i.e., no) category judgments.

The rhyme words were chosen from various rhyming dictionaries. Fourteen words were chosen as "rhyme categories," and 20 words that rhymed with each "category name" were selected. The 280 rhyme words ranged in length from 3 to 8 letters, averaging 5.14 . The rhyme "category names" were sleigh, raid, bear, braille, plane, freight. sign, might, here, plead, scene, tune, too, and though. As in the category task, each rhyme word was paired with a category name it rhymed with for positive (yes) rhyme judgments and with a category name it did not rhyme with for negative (no) rhyme judgments.

The words were displayed on a point-plot CRT (Tektronix Model 604 equipped with P31 phosphor) under the control of a PDP 11/03 laboratory computer. The words were printed in uppercase letters, which were formed by illuminating about 20 points in a $5 \times 7$ dot matrix. Viewed at a distance of $60 \mathrm{~cm}$, each letter subtended $.38 \times .57$ degrees of visual angle. Two words were presented on each trial (i.e., the category name and the word to be judged). The two words were justified four characters to the left of a fixation point in the center of the screen, and the category name appeared one row above the word to be judged.

Each trial began with a fixation point appearing in the center of the screen. After a $.5 \mathrm{~s}$ foreperiod, it was replaced by the word pair for that trial. The word pair was exposed for $1.5 \mathrm{~s}$ whether or not there was a stop signal, except in Experiments 4 and 5 , in which the word pair was extinguished and replaced by two rows of $12 \mathrm{Xs}$ when the stop signal sounded. In Experiments 4 and 5, the $X$ s stayed on for the remainder of the 1.5 -s period that began with the onset of the word pair (i.e., the total duration of the word pair and the rows of $X \mathrm{~s}$ was always $1.5 \mathrm{~s}$, regardless of the stop-signal delay). After the stimulus display was extinguished, a 2-s intertrial interval began.

The stop signal was a $500-\mathrm{ms} 900-\mathrm{Hz}$ tone played through a speaker behind the CRT at a comfortable listening level. In Experiments 4 and 5, the word pair was extinguished and replaced by two rows of $X \mathbf{s}$ when the stop signal sounded, as was described earlier. When the stop signal was presented, it occurred $200,500,800$, or 1,100 $\mathrm{ms}$ after the onset of the word pair. These delays were chosen to provide a range over which subjects would inhibit their responses on about $10 \%$ to $90 \%$ of the stop-signal trials.

Responses were made by pressing one or more of a panel of eight telegraph keys mounted on a movable board in front of the subject. The computer recorded which keys were pressed and the time at which each key was pressed.

\section{Procedure}

Each experiment involved 880 trials divided into eight blocks. Each task was run in four consecutive blocks with half of the subjects completing the category task before the rhyme task and half completing them in the opposite order. Each task involved two kinds of blocks, orienting blocks and repetition blocks. The orienting blocks were 100 trials long and included 50 trials that required positive judgments (yes trials) and 50 trials that required negative judgments ( $n o$ trials). Stop signals were presented on $40 \%$ of the orienting trials, half of the time with yes trials and half of the time with no trials. The repetition blocks were 120 trials long and did not involve the presentation of stop signals. The 120 trials consisted of 40 word pairs that had been presented with stop signals in the immediately preceding orienting block (stop-signal trials), 40 word pairs that had been presented without stop signals in the immediately preceding orienting block (no-signal trials), and 40 word pairs that had not been presented in any preceding orienting block and would not appear in any subsequent orienting or repetition block (new trials). Half of the word pairs of each type demanded a yes response, and half demanded a no response.

Each task involved an orienting block followed by a repetition block and then another orienting block followed by another repetition block. The minimum lag between repetitions of a word pair was 0 trials and the maximum lag was 219 trials (i.e., 100 orienting trials +120 repetition trials - 1). Each subject saw all 280 rhyme words and all 280 category words but the order in which the words were presented, their assignment to response types (yes and no), and their assignment to stop-signal, no-signal, and new trials was randomized separately for each subject.

In each experiment, all subjects pressed the two rightmost keys in the panel of eight to respond to the category and rhyme tasks, using the index and middle fingers of their right hands. Half of the subjects pressed the next-torightmost key for yes responses and the rightmost key for no responses and half did the opposite. The mapping was the same in the orienting trials and in the repetition trials. In Experiments 2 and 3, subjects were required to press the leftmost key with the index fingers of their left hands when the stop signal sounded. 
The instructions described each phase of the procedure as the subject encountered it. Thus, subjects who performed the category task first were not told about the rhyme task until the category task was completed (vice versa for subjects who performed the rhyme task first). In the initial instructions, subjects were told about the relevant task (category or rhyme) and the mapping rules to use in reporting their judgments before they were told about the stopping task. Subjects were told to respond as quickly and accurately as they could on the category and rhyme tasks, but to stop their responses if they could when a stop signal occurred. They were told that stop-signal delays had been selected so that some of the time they would be able to inhibit their responses and some of the time they would not. Subjects were told to treat the category and rhyme tasks as if they were primary and the stopping task (and the overt response to the stop signal in Experiments 2 and 3 ) as if it was secondary.

In the repetition blocks, subjects were told that the task was the same as in the preceding orienting block except that there would be no stop signal. They were not told that some of the word pairs would be repeated. The initial instructions were reviewed briefly for the next orienting block and repetition block. Full instructions were given once again when the task changed (from category to rhyme or vice versa).

\section{Data Analysis}

For the present purposes, the most important data from the repetition blocks are benefit scores, which represent the reduction in reaction time to a word pair the second time it is presented. In general, benefit scores may be defined in two ways: Reaction time to the second presentation of a word pair can be subtracted from reaction time to the first presentation of the same word pair (i.e., orienting reaction time minus repetition reaction time), or reaction time to the second presentation of a word pair can be subtracted from reaction time to the first presentation of a different word pair presented in the same block of trials (i.e., reaction time to new word pairs minus reaction time to stop-signal or no-signal word pairs, all of which are presented in the same repetition block). It was not possible to use the first definition in the present experiments because subjects inhibited more than half of the first-presentation responses to stop-signal trials in the orienting blocks in each experiment.

In calculating benefit scores in the stop-signal conditions, no distinction was made between word pairs whose responses were inhibited and word pairs whose responses failed to be inhibited; stop-signal benefit scores included each word pair that appeared at a particular delay, whether or not the response was inhibited. This procedure was necessary because the comparison between word pairs whose responses were and were not inhibited may be confounded with item-selection effects; at each delay, the slower responses are inhibited and the faster ones escape inhibition (see Logan \& Cowan, 1984). Thus, benefit for inhibited responses should be calculated using a different baseline than benefit for responses that escaped inhibition. However, different baselines could not be used because there was no way to estimate how quickly subjects would have responded had they not inhibited their responses. Thus, stop-signal benefit scores were calculated without attempting to dis- tinguish between responses that were inhibited and responses that were not inhibited. The contrast is made implicitly by comparing stop-signal benefit with no-signal benefit (the former includes more word pairs whose responses were inhibited than the latter) and by evaluating the effects of stop-signal delay (the earlier delays include more word pairs whose responses were inhibited).

Benefit scores were used rather than raw reaction times because raw reaction times include other effects in addition to repetition that could make the analyses cumbersome (e.g., differences in reaction time to new stimuli due to category vs. rhyme task and yes-no response type). The raw reaction times for each experiment are presented in the appendix.

\section{Experiment 1}

In the first experiment, subjects were told to inhibit their responses to the primary (category or rhyme judgment) task when the stop signal sounded. The purpose was to determine whether changing the goal from responding to not responding would inhibit the underlying thoughts. In parallel studies of recognition memory, simply stopping the response did not cause the underlying thoughts to be inhibited (Logan, 1983).

\section{Results and Discussion}

Orienting blocks. For the present purposes, the most important data in the orienting blocks are the probabilities of inhibiting responses when the stop signal sounded. Table 1 presents the complement of these probabilities, the probability of responding to the primary task when a stop signal occurred, presented as a function of stop-signal delay, tasks (category vs. rhyme), and response type (yes vs. no).

On the average, subjects responded on $36.7 \%$ of the stop-signal trials. As in previous stopsignal studies, the probability of responding given a stop signal increased as stop-signal delay increased (see Logan \& Cowan, 1984, for a review). The probability of responding given a signal tended to be higher for yes responses than for no responses but tended to be the same in the category task as in the rhyme task, probably because yes responses tended to be faster than no responses but category judgments were no faster or slower than rhyme judgments (see appendix; also see Logan, 1983; Logan \& Cowan, 1984).

This pattern was confirmed by analysis of variance (ANOVA) on the probabilities of responding given a stop signal: The main effect 
Table 1

Probability of Responding When Given a Stop Signal in the Orienting Task as a Function of Task, Response Type, and Stop-Signal Delay

\begin{tabular}{cccccc}
\hline & & \multicolumn{5}{c}{ Stop-signal delay } \\
\cline { 3 - 6 } Task & Response & 200 & 500 & 800 & 1,100 \\
\hline \multicolumn{5}{c}{ Experiment 1} \\
Category & Yes & .06 & .23 & .58 & .81 \\
& No & .06 & .15 & .40 & .73 \\
Rhyme & Yes & .07 & .17 & .54 & .75 \\
& No & .06 & .12 & .45 & .69 \\
\hline
\end{tabular}

Experiment 2

\begin{tabular}{llllll} 
Category & Yes & .20 & .38 & .74 & .91 \\
& No & .13 & .28 & .66 & .85 \\
Rhyme & Yes & .15 & .23 & .71 & .86 \\
& No & .11 & .23 & .64 & .86 \\
\hline
\end{tabular}

Experiment 4

\begin{tabular}{llllll} 
Category & Yes & .05 & .22 & .59 & .79 \\
& No & .07 & .21 & .47 & .73 \\
Rhyme & Yes & .03 & .16 & .48 & .79 \\
& No & .02 & .15 & .42 & .72 \\
\hline
\end{tabular}

of delay, $F(3,93)=129.58, p<.01, M S_{\mathrm{e}}=$ .020 , and response type, $F(1,31)=39.80, p<$ $.01, M S_{\mathrm{e}}=.015$, were significant, as was the interaction between delay and response type, $F(3,93)=6.14, p<.01, M S_{\mathrm{e}}=.014$.

These results indicate that subjects were able to respond appropriately to the stop signal. The data show effects commonly observed in other stop-signal studies; which suggests that subjects were behaving in similar ways. Thus, conclusions drawn here may be generalized to the earlier studies and vice versa. Furthermore, the response inhibition data fulfill the conditions necessary to evaluate the hypothesis that the underlying thoughts are inhibited with the overt responses: Subjects were more likely to inhibit responses when a stop signal occurred than when no stop signal occurred, and subjects were more likely to inhibit responses the earlier the stop signal occurred. Thus, the underlying thoughts were more likely to have been inhibited on stop-signal trials than on no-signal trials and they were more likely to have been inhibited the earlier the stop signal occurred.

Repetition blocks. The mean benefit scores from stop-signal trials are displayed in Table 2 as a function of stop-signal delay, orienting task (category vs. rhyme), and response type (yes vs. no). The no-signal benefit scores are presented in Table 2 as well. (Recall that benefit scores were calculated in the repetition blocks by subtracting reaction times to stopsignal and no-signal stimuli from reaction times to new stimuli.)

The benefit scores suggest that the underlying thoughts were not inhibited with the overt actions. If the underlying thoughts were inhibited with the overt actions, there should be less benefit on stop-signal trials than on no-signal trials and the benefit on stop-signal trials should increase systematically as stop-signal delay increased. The observed benefit scores showed no such trends. The main effect of stopsignal delay (i.e., 4 delays plus the no-signal condition) was not significant, $F(4,124)<1$, $M S_{\mathrm{e}}=3,702.58$. On the average, there were 43 ms of benefit on stop-signal trials in comparison with $41 \mathrm{~ms}$ of benefit on no-signal trials, a difference that was not significant by a planned comparison, $F(1,124)<1, M S_{\mathrm{e}}=$ $3,702.58$. On the average, there were $38 \mathrm{~ms}$ of benefit at the 200 -ms delay, $45 \mathrm{~ms}$ at the 500 $\mathrm{ms}$ delay, $50 \mathrm{~ms}$ at the $800-\mathrm{ms}$ delay, and 38 $\mathrm{ms}$ at the $1,100-\mathrm{ms}$ delay. A linear trend test assessing the tendency for benefit to increase with stop-signal delay was not significant, $F(1$, 124) $<1, M S_{\mathrm{e}}=3,702.58$. Finally, Fisher's Least Significant Difference (LSD) test was used to compare benefit at each delay with the benefit in the no-signal condition, and found no differences that were significant at the .05 level. Similar results were obtained when the planned comparison, trend test, and LSD tests were repeated in each combination of orienting

Table 2

Benefit From Repetition Priming (in Milliseconds) in Experiment 1 as a Function of Stop-Signal Conditions, Orienting Task, and Response Type

\begin{tabular}{lcccccc}
\hline & & \multicolumn{4}{c}{ Stop-signal delay } & \\
\cline { 3 - 6 } Task & Response & 200 & 500 & 800 & 1,100 & No \\
signal \\
\hline \multirow{2}{*}{ Category } & Yes & 64 & 75 & 95 & 76 & 90 \\
& No & 31 & 43 & 23 & 19 & 24 \\
Rhyme & Yes & 53 & 35 & 63 & 41 & 45 \\
& No & 3 & 25 & 18 & 17 & 4 \\
$M$ & & 38 & 45 & 50 & 38 & 41 \\
\hline \multirow{2}{*}{$M$} & & 38 &
\end{tabular}


task and response type; none of the effects were significant.

These results suggest that the thoughts underlying the category and rhyme judgments ran on to completion ballistically when the overt responses associated with them were inhibited. The present results with repetition priming as the index of thought corroborate earlier results with recognition memory as the index of thought (Logan, 1983, Experiments 1 and 2). Whether thoughts are assessed by repetition priming or recognition memory, simply asking subjects to inhibit the overt responses associated with them does not seem to inhibit the underlying thoughts.

The conditions of the orienting task had large effects on the observed benefit. There was more benefit from category judgments than from rhyme judgments, $F(1,31)=13.10, p<$ $.01, M S_{\mathrm{e}}=6,846.68$, and more benefit from yes responses than from no responses, $F(1$, $31)=37.40, p<.01, M S_{\mathrm{e}}=7,820.29$. The interaction between category versus rhyme and yes versus no was marginally significant, $F(1$, $31)=3.60, p<.10, M S_{\mathrm{e}}=12,831.19$. No other interactions approached significance, including the interactions with stop-signal delay.

\section{Experiment 2}

In Experiment 1, subjects apparently completed the thoughts underlying category and rhyme judgments when they inhibited the overt actions associated with them. Those results could mean that simple thoughts run on to completion ballistically once they begin so that subjects could not have inhibited them if they tried, or the results could mean that subjects deliberately completed the thoughts when they inhibited the associated actions. Indeed, there was nothing in the procedure to prevent them from doing so. Experiment 2 was conducted to determine whether thoughts would still run on to completion when subjects had to switch tasks when the stop signal sounded. The idea was that subjects would be less likely to complete thoughts deliberately if their goal shifted from making category or rhyme judgments to performing a completely different task. Thus, subjects were instructed to respond overtly to the stop signal whenever it sounded (by pressing a separate key) in addition to in- hibiting their response to the category or rhyme task.

\section{Results and Discussion}

Orienting blocks. The probability of responding to the word pair when the stop signal sounded is presented in Table 1 for each combination of conditions (category vs. rhyme judgments; yes vs. no responses) as a function of stop-signal delay. On the average, subjects responded on $49.6 \%$ of the stop-signal trials. As in the previous study, the probability of responding given a stop signal increased as stop-signal delay increased, was higher for yes responses than for no responses, but was about the same for category judgments as for rhyme judgments.

These conclusions were confirmed by an ANOVA: The main effects of delay, $F(3,93)=$ $219.56, p<.01, M S_{\mathrm{e}}=.065$, and response type, $F(1,31)=53.10, p<.01, M S_{\mathrm{e}}=.010$, were significant, but the main effect of orienting task was not, $F(1,31)<1, M S_{\mathrm{e}}=.179$. The interaction between delay and response type was marginally significant, $F(3,93)=$ $2.59, p<.06, M S_{\mathrm{e}}=.010$. The reaction times for the overt response to the stop signal appear in the appendix.

The inhibition data indicate that subjects were able to respond appropriately to the stop signal, reflecting trends observed in previous studies. Moreover, they fulfill the conditions necessary to test the hypothesis that the underlying thoughts are inhibited with the overt actions: Subjects inhibited responses more often with a stop signal than without one, and they inhibited responses more often the earlier the stop signal occurred. Thus, the underlying thoughts were more likely to be inhibited with a stop signal than without one and were more likely to be inhibited the earlier the stop-signal delay.

Repetition blocks. The mean benefit scores from stop-signal trials are displayed in Table 3 as a function of stop-signal delay, orienting task, and response type. The benefit scores from no-signal trials are displayed in Table 3 as well.

The benefit scores show small differences between stop-signal and no-signal trials and weak effects of stop-signal delay. The main effect of stop-signal delay was not significant, 
Table 3

Benefit From Repetition Priming (in Milliseconds) in Experiment 2 as a Function of Stop-Signal Conditions, Orienting Task, and Response Type

\begin{tabular}{lcrrrrr} 
& & \multicolumn{4}{c}{ Stop-signal delay } & \\
\cline { 3 - 6 } Task & Response & 200 & 500 & 800 & 1,100 & so \\
signal \\
\hline \multirow{2}{*}{ Category } & Yes & 59 & 87 & 90 & 71 & 92 \\
& No & 26 & 38 & 42 & 51 & 48 \\
Rhyme & Yes & 30 & 36 & 30 & 51 & 47 \\
& No & 14 & 7 & 9 & 6 & 26 \\
$M$ & & 32 & 42 & 42 & 45 & 53 \\
\hline
\end{tabular}

$F(4,124)=1.72, M S_{\mathrm{e}}=4,010.88$. On the average, there were $40 \mathrm{~ms}$ of benefit on stop-signal trials, in comparison with $53 \mathrm{~ms}$ of benefit on no-signal trials; a planned comparison assessing the difference was significant, $F(1$, $124)=4.12, p<.05, M S_{\mathrm{e}}=4,010.88$. On the average, there were $32 \mathrm{~ms}$ of benefit at the 200$\mathrm{ms}$ delay, $43 \mathrm{~ms}$ at the $500-\mathrm{ms}$ delay, $42 \mathrm{~ms}$ at the 800 -ms delay, and $45 \mathrm{~ms}$ at the $1,100 \mathrm{~ms}$ delay, but the linear trend assessing the tendency for benefit to increase with stop-signal delay was not significant, $F(1,124)=2.12$, $M S_{\mathrm{e}}=4,010.88$. Fisher's LSD test was used to compare the benefit at each stop-signal delay with the no-signal benefit, and found a significant difference at the 200 -ms delay $(p<.05)$. Fisher's LSD test also showed that the benefit at each stop-signal delay was significantly greater than zero $(p<.01)$.

The planned comparison, linear trend, and LSD analyses were repeated within each combination of orienting task and response type, revealing a difference that was significant by the LSD test $(p<.05)$ between the 200 -ms delay and the no-signal condition for yes responses in the category task. The benefit scores in each combination of conditions were significantly greater than zero $(p<.05)$, except for the $200-\mathrm{ms}$ delay in the category-no condition and all of the delays in the rhyme-no condition, including the no-signal data.

These results suggest that switching the goal from responding to the word pair to responding to the tone inhibited the thoughts underlying the category and rhyme judgments at least partially; there was less benefit with a stop signal than without one, and there was a tendency for there to be less benefit the shorter the stop- signal delay. However, the results also indicate that switching the goal did not inhibit thoughts entirely; benefit was significantly greater than zero in most stop-signal conditions. Together, these effects suggest that switching goals from one task to another has a weak inhibitory effect on the thoughts underlying performance on the first task. A similar conclusion was reached in parallel studies with recognition memory as the index of inhibited thought (Logan, 1983, Experiments 3 and 4).

One possibility is that the overt response to the tone interfered with thought whether or not subjects managed to inhibit their response to the word pair. This was apparent in the earlier studies of recognition memory, in which the requirement to respond to the tone depressed subsequent memory performance for words whose responses were not inhibited as much as it did for words whose responses were inhibited (Logan, 1983, Experiments 3 and 4). In the present experiment, it was not possible to compare repetition priming for word pairs whose responses were and were not inhibited because there was no appropriate baseline against which to assess benefit for word pairs whose responses were inhibited (see above). Instead, Experiment $\mathbf{3}$ was conducted, in which subjects responded overtly to the tones without inhibiting their responses to the word pairs. If the depressed repetition priming in the present experiment was due to the requirement to respond overtly to the tone, then the same depression of repetition priming should be observed in Experiment 3. However, if the depressed repetition priming in the present experiment was due to inhibition of thought on those trials on which the response was inhibited, then there should be no depression of repetition priming in Experiment 3.

The results of Experiment 2 also bear on the interpretation of Experiment 1, where the null effects of stop-signal versus no-signal conditions and the null effect of stop-signal delay could mean that the underlying thoughts were completed ballistically, without intention, or deliberately, to alleviate boredom or to satisfy curiosity. The switch to the new task in Experiment 2 should have reduced the tendency to complete thoughts deliberately, so the results should be substantially different from those in Experiment 1 if subjects in Experiment 1 completed their thoughts deliberately. The re- 
sults were different, but the differences were not very large. Thus, it does not seem very likely that subjects in Experiment 1 completed their thoughts deliberately when they inhibited the associated action.

Orienting task conditions again had large effects on the observed benefit. Category judgments produced significantly more benefit than rhyme judgments, $F(1,31)=9.98, p<$ $.01, M S_{\mathrm{e}}=18,840.77$, and yes responses produced significantly more benefit than no responses, $F(1,31)=30.32, p<.01, M S_{\mathrm{e}}=$ $5,529.81$. The interaction between them was not significant, nor were any other interactions in the analysis, including the interactions with stop-signal delay.

\section{Experiment 3}

Experiment 3 was conducted as a control for Experiment 2. Its purpose was to determine whether having to respond overtly to a tone (i.e., the stop signal) during the orienting blocks would affect the amount of repetition priming observed subsequently in the repetition blocks even if subjects completed the response to the orienting task. Experiment 3 was an exact replication of Experiment 2, except that subjects were not told to inhibit their responses when the stop signal occurred. They were told to respond overtly to the word pair on every trial and to respond overtly to the stop signal when it occurred.

\section{Results and Discussion}

Orienting blocks. Subjects were told to respond to the word pair on every trial, so there were no inhibition data to report. The mean reaction times to the word pairs and the mean reaction times for the overt responses to the tone are presented in the appendix.

Repetition blocks. The mean benefit scores from tone trials are displayed in Table 4 as a function of tone delay, orienting task, and response type. The mean benefit scores from notone trials also appear in Table 4.

The requirement to respond overtly to the tone had little effect on performance. The main effect of tone delay was not significant, $F(1$, 124) $<1, M S_{\mathrm{e}}=4,325.77$. Overall, the mean benefit on tone trials was $40 \mathrm{~ms}$ in comparison
Table 4

Benefit From Repetition Priming (in Milliseconds) in Experiment 3 as a Function of Tone Conditions, Orienting Task, and Response Type

\begin{tabular}{lcrrrrr}
\hline & & \multicolumn{4}{c}{ Tone-delay } & \\
\cline { 3 - 6 } Task & Response & 200 & 500 & 800 & 1,100 & No \\
tone \\
\hline Category & Yes & 68 & 64 & 84 & 76 & 68 \\
& No & 16 & 39 & 32 & 17 & 35 \\
Rhyme & Yes & 40 & 20 & 45 & 68 & 39 \\
& No & 33 & 8 & 22 & 3 & 17 \\
$M$ & & 39 & 33 & 46 & 41 & 40 \\
\hline
\end{tabular}

with a mean benefit of $40 \mathrm{~ms}$ of benefit on notone trials. A planned comparison evaluating this difference was not significant, $F(1,124)<$ $1, M S_{\mathrm{e}}=4,325.77$. On the average, there were $39 \mathrm{~ms}$ of benefit at the $200-\mathrm{ms}$ delay, $33 \mathrm{~ms}$ at the 500 -ms delay, $46 \mathrm{~ms}$ at the 800 -ms delay, and $41 \mathrm{~ms}$ at the $1,100-\mathrm{ms}$ delay. A linear trend test assessing the tendency for benefit to increase with tone delay was not significant, $F(1$, 124) $<1, M S_{\mathrm{e}}=4,325.77$. None of the tonetrial benefit scores was different from the notone score by Fisher's LSD test $(p<.05)$. These analyses were repeated in each combination of orienting task and response type, and revealed no significant effects.

These results suggest that the requirement to perform a concurrent task during the orienting trials had little effect on the amount of repetition priming observed. Completing the underlying thought seems to produce the same amount of repetition priming whether or not subjects engage in another task concurrently. These results contrast with the findings in Experiment 2, where stopping the overt response to the word pair while responding to the tone tended to inhibit the underlying thoughts. An ANOVA was conducted to evaluate this contrast formally: The interaction between experiments (Experiment 2 vs. Experiment 3) and tone-delay conditions was not significant, $F(4,248)<$ $1, M S_{e}=5,938.44$, but a planned contrast that compared the difference between stop-signal and no-signal benefit in Experiment 2 with the difference between stop-signal and no-signal benefit in Experiment 3 was significant, $F(1$, $248)=3.97, p<.05$, indicating that the difference was larger in Experiment 2 than in Experiment 3. 
Orienting task conditions had strong effects on the amount of repetition priming in Experiment 3: There was more benefit following category judgments than following rhyme judgments, $F(1,31)=5.90, p<.05, M S_{\mathrm{e}}=$ $11,354.71$, and there was more benefit following yes responses than following no responses, $F(1,31)=16.84, p<.01, M S_{\mathrm{e}}=11,679.26$. The interaction between them was not significant, $F(1,31)=2.03, p<.20, M S_{\mathrm{e}}=$ $6,487.13$, nor was any other interaction in the analysis, including the interactions with tone delay.

\section{Experiment 4}

Experiments 1 and 2 suggested that neither simply inhibiting the overt response nor inhibiting the response and switching to a new task had strong inhibitory effects on the simple thoughts underlying category and rhyme judgments. Experiment 4 was conducted to determine whether terminating the display and replacing it with another one (two strings of $X \mathrm{~s}$ ) would be sufficient to inhibit thought. Parallel studies with recognition memory as the index of thought suggested that changing the display would inhibit thought, perhaps because the stimuli that drive the thoughts would no longer be present (Logan, 1983, Experiments 5 and 6).

\section{Results and Discussion}

Orienting blocks. The probability of responding to the word pair when a stop signal sounded and the display changed are presented earlier in Table 1 for each combination of stopsignal delay, orienting task, and response type. On the average, subjects responded on $36.9 \%$ of the stop-signal trials. As before, the probability of responding given a stop signal increased as stop-signal delay increased, was higher for yes responses than for no responses, and was not much different for category judgments than for rhyme judgments.

These effects were confirmed by an ANOVA. The main effects of stop-signal delay, $F(3$, $93)=166.26, p<.01, M S_{\mathrm{e}}=.078$, and response type, $F(1,31)=25.07, p<.01$, $M S_{\mathrm{e}}=.008$, were significant, as was the interaction between delay and response type, $F(3$, 93) $=5.84, p<.01, M S_{\mathrm{e}}=.011$. The main
Table 5

Benefit From Repetition Priming (in Milliseconds) in Experiment 4 as a Function of Stop-Signal Conditions, Orienting Task, and Response Type

\begin{tabular}{lcrrrrr}
\hline & & \multicolumn{4}{c}{ Stop-signal delay } & \\
\cline { 3 - 6 } Task & Response & 200 & 500 & 800 & 1,100 & No \\
signal \\
\hline \multirow{2}{*}{ Category } & Yes & 23 & 54 & 75 & 83 & 92 \\
\multirow{3}{*}{ Rhyme } & No & 9 & 29 & 49 & 31 & 43 \\
& Yes & 29 & 33 & 53 & 46 & 37 \\
& No & 1 & 5 & 3 & 44 & 37 \\
$M$ & & 15 & 30 & 45 & 51 & 52 \\
\hline
\end{tabular}

effect of orienting task was marginally significant, $F(1,31)=3.62, p<.07, M S_{\mathrm{c}}=.065$.

Once again, these effects suggest that subjects were able to respond appropriately to the stop signal, so the results should generalize to other studies. Moreover, the data fulfill the conditions necessary to evaluate the hypothesis that the underlying thoughts are inhibited with the overt actions: Subjects were more likely to inhibit their responses on stop-signal trials than on no-signal trials, and they were more likely to inhibit their responses the earlier the stop signal occurred.

Repetition blocks. The mean benefit scores from stop-signal trials are displayed in Table 5 as a function of stop-signal delay, orienting task, and response type. The mean benefit scores from no-signal trials also appear in $\mathrm{Ta}$ ble 5 .

In this experiment, the benefit scores suggest that thoughts were inhibited with the overt actions: The main effect of stop-signal delay was highly significant, $F(1,124)=5.66, p<.01$, $M S_{\mathrm{e}}=5,621.86$. On the average, there were $35 \mathrm{~ms}$ of benefit on the stop-signal trials in comparison with 52 ms of benefit on the nosignal trials, and the difference was significant, $F(1,124)=5.22, p<.05, M S_{\mathrm{e}}=5,621.86$. Benefit increased substantially as stop-signal delay increased: There were $15 \mathrm{~ms}$ of benefit at the 200 -ms delay, $30 \mathrm{~ms}$ at the 500 -ms delay, $45 \mathrm{~ms}$ at the $800-\mathrm{ms}$ delay, and $51 \mathrm{~ms}$ at the 1,100-ms delay. The linear trend test assessing the tendency for benefit to increase with delay was significant, $F(1,124)=16.91, p<.01$, $M S_{\mathrm{e}}=5,621.86$. Fisher's LSD test showed that the benefit in the no-signal condition was significantly larger than the benefit at the $200-\mathrm{ms}$ 
delay $(p<.01)$ and the benefit at the 500-ms delay $(p<.05)$. It also showed that the benefit at the 200-ms delay was not significantly greater than zero, but the benefit scores at the longer delays were all significantly greater than zero $(p<.05)$.

The planned comparison, trend test, and LSD test were repeated in each combination of orienting task and response type. The mean of the stop-signal conditions was significantly different from the no-signal condition for yes responses in the category task, $F(1,124)=$ $5.03, p<.05, M S_{\mathrm{e}}=5,702.05$; the linear trend was significant for yes responses in the category task, $F(1,124)=11.30, p<.01, M S_{\mathrm{e}}=$ $5,702.05$, and for no responses in the rhyme task, $F(1,124)=4.58, p<.05, M S_{e}=$ 5,702.05; and Fisher's LSD test revealed significant differences between the 200-ms delay and the no-signal condition $(p<.01)$ and between the 500-ms delay and the no-signal condition $(p<.05)$ for yes responses in the category task. According to Fisher's LSD test, all benefit scores greater than 37 ms were significantly greater than zero $(p<.05)$. Nine out of 16 benefit scores in the stop-signal conditions were not significantly greater than zero.

These results are not consistent with the hypothesis that thoughts ran on to completion ballistically when the stop signal sounded and the display changed. There was less benefit with a stop-signal than without one, and the benefit with a stop-signal increased strongly as stopsignal delay increased. Moreover, the benefit at the shortest delays was not significantly greater than zero (i.e., chance). This suggests that, as in the parallel studies of recognition memory (Logan, 1983, Experiments 5 and 6), terminating the display and replacing it with a new one is sufficient to inhibit the thoughts underlying category and rhyme judgments.

Orienting block conditions had substantial effects on the observed benefit once again. There was more benefit following category judgments than following rhyme judgments, $F(1,31)=4.05, p<.06, M S_{\mathrm{e}}=15,871.29$, and there was more benefit following yes responses than following no responses, $F(1,31)=$ $12.88, p<.01, M S_{\mathrm{e}}=9,479.74$. The interaction between them was not significant, $F(1$, 31) $<1, M S_{\mathrm{e}}=15,401.65$, nor was any other interaction in the analysis, including the interactions with stop-signal delay.

\section{Experiment 5}

Experiment 5 was conducted as a control for Experiment 4, to determine whether the reduction in benefit at the early stop-signal delays was due to the inhibition of thought or to a general depression of performance due to masking. Experiment 5 was an exact replication of Experiment 4, except that subjects were not told to inhibit their responses when the tone sounded and the display changed. They were told to complete their responses to the word pairs on each trial, whether or not the display changed. If the reduction in benefit in Experiment 4 was due to the inhibition of thought, there should be no reduction here because subjects were told to deliberately complete their thoughts in all conditions. However, if the reduction in benefit in Experiment 4 was due to masking from the new display, then the same reduction in benefit should be observed in this experiment.

\section{Results and Discussion}

Orienting blocks. There were no inhibition data to report because subjects were instructed to respond on every trial. The mean reaction times in each orienting condition are reported in the appendix.

Repetition blocks. The mean benefit scores from display-change trials are presented in Table 6 as a function of stop-signal delay, orienting task, and response type. The mean benefit scores from no-change trials are also presented in Table 6.

The data in Table 6 suggest that changing the display had a weak effect on the amount of repetition priming. The main effect of display-change delay was not significant, $F(4$, 124) $=1.91, M S_{\mathfrak{e}}=6,255.03$. Overall, there were $42 \mathrm{~ms}$ of benefit in the display-change conditions, in comparison with $43 \mathrm{~ms}$ in the no-change condition, $F(1,124)<1, M S_{\mathrm{e}}=$ $6,255.03$. There were $25 \mathrm{~ms}$ of benefit at the 200-ms delay, $45 \mathrm{~ms}$ at the $500-\mathrm{ms}$ delay, 46 ms at the 800 -ms delay, and $50 \mathrm{~ms}$ at the $1,100-$ ms delay; the linear trend test assessing the tendency for benefit to increase with displaychange delay was significant, $F(1,124)=5.77$, $p<.05, M S_{\mathrm{e}}=6,255.03$. Fisher's LSD test showed that none of the display-change conditions were significantly different from the no- 
Table 6

Benefit From Repetition Priming in (in Milliseconds) Experiment 5 as a Function of Display-Change Conditions, Orienting Task, and Response Type

\begin{tabular}{ccrrrrr}
\hline & & \multicolumn{4}{c}{ Display-change delay } & \\
\cline { 3 - 6 } Task & Response & 200 & 500 & 800 & 1,100 & No \\
change
\end{tabular}

change condition $(p<.05)$, and that every one of the display-change conditions was significantly greater than zero $(p<.05)$.

These analyses were repeated for each combination of orienting tasks and response type, revealing a significant linear increase in benefit with delay for no responses in the category task, $F(1,124)=4.31, p<.05, M S_{\mathrm{e}}=6.830 .69$, and a difference between the benefit at the 200$m s$ delay and the benefit in the no-change condition for yes responses in the rhyme task that was significant by Fisher's LSD test $(p<.05)$.

Overall, these results suggest that changing the display without inhibiting the required responses had a small inhibitory influence on the underlying thoughts: There was a tendency for benefit to increase with stop-signal delay that was significant in the overall analysis and was significant in some conditions when conditions were analysed separately. The pattern of results is similar to the pattern in Experiment 4 , in which subjects had to inhibit their responses when the display changed, but the inhibitory effects were not as strong. The contrast between Experiments 4 and 5 was assessed formally by analysing both experiments in one ANOVA: The interaction between experiments and delay conditions was not significant, $F(4$, $248)=1.10, M S_{\mathrm{e}}=4,167.66$, but a planned comparison showed that the difference in benefit between the no-change condition and the mean of the display-change conditions was significantly larger in Experiment 4 than it was in Experiment $5, F(1,248)=3.89, p<.05$. These results suggest that terminating the display and replacing it with a new one can inhibit thought somewhat, but the inhibitory effect is stronger if the subject's goal changes (from responding to not responding) when the display changes.

Again, orienting block conditions affected the observed benefit. There was more benefit following category judgments than following rhyme judgments, $F(1,31)=4.69, p<.05$, $M S_{\mathrm{e}}=44,273.97$, and there tended to be more benefit following yes responses than following no responses, $F(1,31)=3.52, p<.07, M S_{\mathrm{e}}=$ $29,267.74$. The interaction between them was not significant, nor was any other interaction in the analysis, including the interactions with display-change delay.

\section{General Discussion}

Five experiments were conducted to investigate the ability to control thoughts that are no longer relevant to current goals, which is presumably an important aspect of cognitive control. In each experiment, subjects performed an orienting phase in which their goal was to make category and rhyme judgments about word pairs, and occasionally, they were presented with a stop signal that told them to change their goal and inhibit the overt response to the word pair on that trial. The extent to which the underlying thought was inhibited when the goal changed was assessed by presenting the word pairs again in a repetition phase and comparing the amount of repetition priming for word pairs whose responses were inhibited (stop-signal word pairs) with the amount for word pairs whose responses were not inhibited (no-signal word pairs). Thoughts about no-signal word pairs were likely to have gone on to completion because the corresponding responses were made with a high level of accuracy. If the thoughts about stop-signal word pairs were inhibited with the responses, those word pairs should produce less repetition priming than no-signal word pairs and there should be less repetition priming the shorter the stop-signal delay. If the thoughts about stopsignal word pairs ran on to completion nonetheless, then those word pairs should produce the same amount of repetition priming as nosignal word pairs and the amount of repetition priming should be independent of stop-signal delay.

Experiment 1 assessed the inhibition of thought in a situation in which subjects were 
asked to simply inhibit their responses when the stop signal sounded. Its purpose was to see whether simply changing the goal from responding to not responding was sufficient to inhibit the underlying thought. Apparently it was not sufficient. Word pairs presented with a stop signal produced as much benefit as word pairs presented without a stop signal, and the benefit for stop-signal word pairs was not affected by stop-signal delay; the early delays where responses were inhibited most often produced as much benefit as the late delays where responses were inhibited least often.

Experiment 2 assessed the inhibition of thought in a situation in which subjects switched to a new task (responding overtly to the stop signal) when they inhibited their responses to the category or rhyme task. Its purpose was to see whether changing the goal from performing one task to performing another and consequently mobilizing resources to respond to the new goal would inhibit the thoughts underlying the original task. The results suggested that switching to a new task had a weak inhibitory effect on the thoughts underlying the first task: On the one hand, there was significantly less benefit with a stop signal than without one and there tended to be less benefit at the earliest stop-signal delay, which suggests that the thoughts were inhibited at least partially. On the other hand, benefit in most conditions was significantly greater than zero, which suggests that the thoughts were not inhibited completely.

Experiment 3 was conducted as a control for Experiment 2, to determine whether adding a new task without abandonning the old one would affect the underlying thoughts. Subjects responded overtly to the tone when it occurred without inhibiting their responses to the word pairs. The results suggested that the requirement to respond to the tone concurrently had little effect on the amount of repetition priming. Word pairs presented with a tone produced as much benefit as word pairs presented without one, and the benefit from word pairs presented with a tone was not affected by tone delay.

Experiment 4 assessed the inhibition of thought in a situation in which the stimuli that drive the thoughts were removed and replaced with neutral stimuli when the stop signal sounded. Its purpose was to determine whether thoughts would still go on to completion when there was no goal and no stimuli to drive them. The results suggested that the thoughts were inhibited. Stop-signal word pairs produced less benefit than no-signal word pairs, and the benefit from stop-signal word pairs increased substantially as stop-signal delay increased. Moreover, the benefit at the early delays was often not different from zero (i.e., chance). These results suggested that thoughts may not run on to completion without a goal and a stimulus to drive them.

Experiment 5 was conducted as a control for Experiment 4, to determine whether the reduced benefit observed in Experiment 4 was due to the inhibition of thought or to a masking effect from the stimulus that replaced the word pair. The display changed on occasion during the orienting trials, just as it did in Experiment 4 , but subjects were instructed to respond on every trial, whether or not the display changed. Thus, the goal stayed the same when the display changed. The results suggested that the amount of repetition priming was not affected much by the change in the display. On the one hand, there was a tendency for benefit to increase as the delay of the change in the display increased, but on the other hand, word pairs whose displays were changed produced about as much benefit as word pairs whose displays were not changed and, averaged over orienting conditions, the benefit at each change delay was significantly greater than zero.

These experiments suggest that thoughts that are no longer relevant to current goals may still run on to completion (e.g., Experiment 1) unless subjects switch to a new task (Experiment 2) or the stimuli that drive the thoughts are removed (Experiment 4). There does not appear to be any deliberate inhibition of thought; instead, the thoughts appear to be "crowded out" or suppressed when thoughts that are relevant to the new task or the new stimulus take their place. This may be sufficient for cognitive control in many task environments: A change in goals often engenders switching to a new task and attending to new stimulation, so the task environment may provide the conditions that are necessary and sufficient to inhibit the old thoughts that are no longer relevant. Moreover, the experiments showed that relatively simple thoughts could be inhibited by switching tasks and changing 
stimulation. In view of this, it seems unlikely that complex chains of thought would carry on for long when they were no longer relevant. Deliberate inhibition does not seem to be necessary.

Note that the present experiments were concerned with the ability to inhibit simple thoughts after they began and with the relation between the inhibition of thought and the inhibition of action. Thus, stop-signal delays were selected so that subjects would likely have time to begin thinking about the word pairs before the signal sounded and so that the probability of inhibiting responses would vary over a large range (i.e., from .10 to .90). The data suggest that the delays I selected were successful in these respects. It is possible, however, that a different picture would emerge if very short delays were used: In Experiment 2, for example, repetition priming began to decrease below the level on no-signal trials at the shortest delay ( $200 \mathrm{~ms})$, though it was still greater than zero; if the stop signal occurred much earlier (i.e., before the word pair appeared), repetition priming may have been eliminated entirely. If the stop signal occurs early enough, subjects may never begin to think about the word pair, so there may be no "aftereffect" of thought to produce repetition priming. Alternatively, subjects may not be able to stop themselves from thinking about the word pair no matter how early the stop signal is presented; the simple thoughts investigated in these experiments may begin entirely without intention. That is an important question for future research. However, it is different from the question addressed by the present experiments, which is whether thoughts can run on to completion without intention after they begin.

\section{Repetition Priming and Recognition as Measures of Memory}

Thought inhibition effects. The present experiments were designed to replicate aspects of previous stop-signal experiments that used recognition memory as an index of thought (Logan, 1983). They used the same materials and displayed them on the same apparatus under very similar conditions. The idea was to see whether a different measure of memory would lead to the same conclusions. Interest- ingly, the major manipulations had similar effects, though repetition priming seemed more sensitive than recognition memory as an index of thought inhibition. Simply changing the goal from responding to not responding had little impact on the underlying thoughts, whether they were assessed by repetition priming or by recognition memory. This can be seen by comparing the effect of stop-signal delay on the benefit scores in the present Experiment 1 with the effect of stop-signal delay on recognition hit rates in Logan's (1983) Experiment 2 . The data are presented in the top two panels of Figure 1. In both cases, stop signal delay had little effect on memory performance, and memory performance on stop-signal trials was not much different from memory performance on no-signal trials.

Changing the goal from responding to one task to responding to another had little impact on the underlying thoughts as assessed by recognition memory (Logan, 1983, Experiment 3) but it had a weak inhibitory effect on the underlying thoughts as assessed by repetition priming (the present Experiment 2). In both cases, the requirement to stop and respond to the tone depressed performance, relative to nosignal trials, but stop-signal delay had very little effect on performance (see the middle two panels of Figure 1). However, changing the goal and changing the display had a strong inhibitory effect, whether thoughts were assessed by repetition priming (the present Experiment 4) or by recognition memory (Logan, 1983, Experiment 6). Repetition priming and recognition hit rates both increased as stop-signal delay increased, and both were smaller on stopsignal trials than on no-signal trials (see the bottom two panels of Figure 1).

The fact that two different measures of memory performance produce similar results should enhance our confidence in the conclusions. This is especially true because repetition priming and recognition memory probably reflect different aspects of memory, either different memory systems (Tulving et al., 1982) or different information in the same memory system (Jacoby, 1983).

Levels of processing effects. Orienting task conditions had significant effects in each of the present experiments. Category judgments consistently produced more repetition priming than rhyme judgments did, and yes responses 

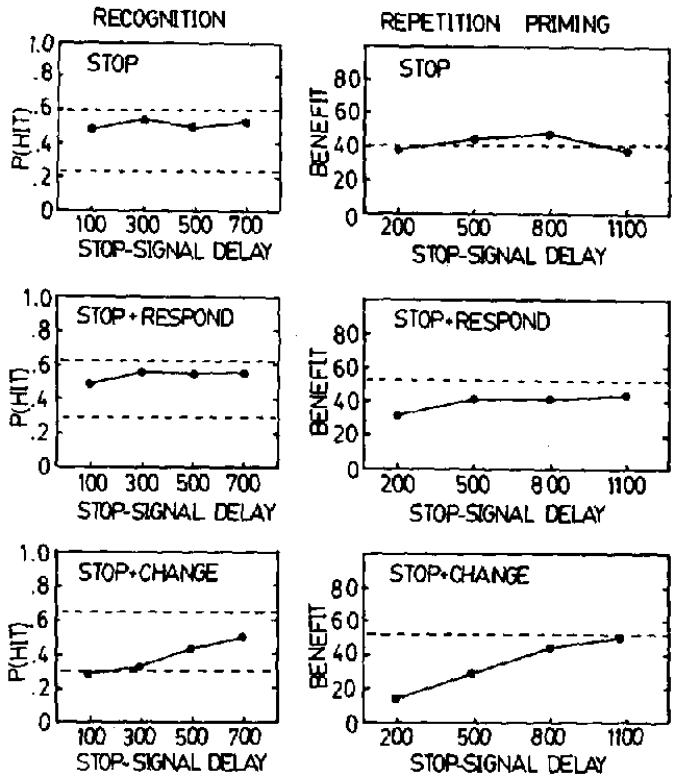

Figure 1. Inhibition of thought as assessed from recognition memory (left-hand panels; from Logan, 1983) and from repetition priming (right-hand panels; from the present experiments) as a function of stop-signal delay, averaged over orienting tasks and response type. (The top horizontal line in each of the left-hand panels and the single horizontal line in each of the right-hand panels reflect performancehit rate or benefit from repetition-on no-signal trials. The bottom horizontal line in each of the left-hand panels reflects the mean false alarm rate. The top two panels reflect performance when subjects are told only to stop when the signal sounds; the top left panel is from Experiment 2 of Logan, 1983, and the top right panel is from the present Experiment 1 . The middle two panels reflect performance when subjects must stop their response to the primary task and respond overtly to the stop signal when it sounds; the middle left panel is from Experiment 3 of Logan, 1983, and the middle right panel is from the present Experiment 2 . The bottom two panels reflect performance when subjects simply stop their responses but the display changes when the stop signal sounds; the bottom left panel is from Experiment 6 of Logan, 1983, and the bottom right panel is from the present Experiment 4.)

consistently produced more repetition priming than no responses did. Effects like these are often found with recognition or recall as measures of memory (e.g., Logan, 1983) but they have not been found previously in tests of repetition priming. For example, Jacoby and Dallas (1981) found no greater benefit in a subsequent perceptual identification test for words that were presented first in a semantic orienting task (in which subjects answered questions about the words' meaning) than for words that were presented first in a rhyme orienting task. The lack of levels of processing effects in their perceptual identification task led Jacoby and Dallas and others to suggest a dissociation between repetition priming and other tests of memory. The present experiments suggest that such a dissociation need not always be found (also see Jacoby, 1983).

An important difference between the present studies and the Jacoby and Dallas (1981) studies is in the nature of the task with which repetition priming was assessed. Jacoby and Dallas used the same task (perceptual identification) to assess repetition priming for both of their orienting tasks, whereas the present studies used a different task for each orienting task (i.e., a category task to assess benefit from prior categorization and a rhyme task to assess benefit from prior rhyming). This difference may have been responsible for the difference in results.

It could be argued that it is more appropriate to assess repetition priming on a common task, as Jacoby and Dallas did, than to assess it from different tasks, as I did. This would certainly be true if the different tasks were selected arbitrarily with no obvious relation to the task used in the initial presentation. However, my choice of tasks is appropriate if we consider repetition priming as a form of learning. From that perspective, it would be appropriate to assess benefit on a second presentation with the same task that was used on the first presentation, to assess subjects' learning of the task. Thus, the present results suggest that subjects may learn more about categorization from one exposure to a categorization task than they learn about rhyming from one exposure to a rhyming task (cf. Morris, Bransford, \& Franks, 1977).

Jacoby (1983; Jacoby \& Brooks, 1984) took a similar position, arguing that benefit from repetition priming depends on the similarity of the original task to the repetition task. Cases like the present experiments, in which the original task is identical to the repetition task, should produce maximal transfer, and thus, maximal repetition priming.

Note that the present results are not subject to an alternative interpretation, that the levels of processing effects reflect differences in initial performance rather than differences in transfer. 
Often, category judgments are slower than rhyme judgments, so there is more room for improvement with category judgments than with rhyme judgments. However, in the present experiments, category judgments were not slower than rhyme judgments on the initial presentation, so there was no more room for improvement with category judgments than with rhyme judgments (see appendix; also see Logan, 1983). The difference in benefit occurred because category judgments were faster than rhyme judgments on the second presentation. Note as well that this argument cannot account for the increased benefit for yes responses relative to no responses because yes responses were faster than no responses even on the first presentation. If anything, there was less room for improvement for yes responses than for no responses, yet yes responses showed more benefit from repetition.

The levels of processing effects suggest that repetition priming may be an associative phenomenon: The amount of repetition priming depends on more than the mere presentation of a word; it also depends on the context in which the word appeared. Words presented with category names produced more priming than words presented with words they rhymed with, and words presented in congruent pairs (which led to yes responses) produced more priming than words presented with incongruent pairs (which led to no responses). Thus, repetition priming may depend on memory for the association between the words in a pair rather than memory for each word separately. By contrast, Jacoby and Dallas (1981) and Feustel, Shiffrin, and Salasoo (1983) argue that repetition affects the ability to encode stimuli, which may be interpreted as a nonassociative effect. Possibly, repetition priming depends on both associative and nonassociative effects. More research is needed to clarify the issue.

The present experiments rule out one possible associative basis for repetition priming, associations between stimuli and overt responses. Experiment 1 demonstrated that the amount of repetition priming is about the same whether or not the subject executes an overt response (i.e., there was little difference between stop-signal and no-signal trials and no effect of stop-signal delay). Thus, stimulus-re- sponse associations cannot be an important basis of the repetition effect.

\section{Conclusions}

The present experiments suggest that the simple thoughts underlying category and rhyme judgments tend to run on to completion ballistically when the overt responses associated with them are inhibited unless subjects switch to a new task or the stimuli that drive the thoughts are removed and replaced by neutral stimuli. The results are consistent with previous stop-signal studies that used recognition memory as the index of inhibited thought, which may reflect different aspects of thought. Thus, the conclusions appear to generalize beyond a single index of thought.

\section{References}

Battig, W. F., \& Montague, W. E. (1969). Category norms for items in 56 categories: A replication and extension of the Connecticut Category Norms. Journal of Experimental Psychology Monograph, 80, (3, Pt. 2).

Feustel, T. C., Shiffrin, R. M., \& Salasoo, A. (1983). Episodic and lexical contributions to the repetition effect in word identification. Journal of Experimental Psychology: General, 112, 309-346.

Jacoby, L. L. (1983). Perceptual enhancement: Persistent effects of an exposure. Journal of Experimental Psychology: Learning, Memory, and Cognition, 9, 21-38.

Jacoby, L. L., \& Brooks, L. R. (1984). Nonanalytic cognition: Memory, perception, and concept learning. In G. H. Bower (Ed.), The psychology of learning and motivation: Advances in research and theory (Vol. 18). New York: Academic Press.

Jacoby, L. L., \& Dallas, M. (1981). On the relationship between autobiographical memory and perceptual learning. Journal of Experimental Psychology: General. $110,306-340$.

Logan, G. D. (1983). On the ability to inhibit simple thoughts and actions: I. Stop-signal studies of decision and memory. Journal of Experimental Psychology: Learning, Memory, and Cognition, 9, 585-606.

Logan, G. D., \& Cowan, W. B. (1984). On the ability to inhibit thought and action: A theory of an act of control. Psychological Review, 91, 295-327.

Morris, C. D., Bransford, J. D., \& Franks, J. J. (1977). Levels of processing versus transfer appropriate processing. Journal of Verbal Learning and Verbal Behavior. 16, 519-533.

Scarborough, D., Cortese, C., \& Scarborough, H. (1977). Frequency and repetition effects in lexical memory. Journal of Experimental Psychology: Human Perception and Performance, 3, 1-17.

Tulving, E., Schacter, D. L., \& Stark, H. (1982). Priming effects in word-fragment completion are independent of recognition memory. Journal of Experimental Psychotogy: Learning, Memory, and Cognition, 8, 336-342. 
Appendix

Table A1

Mean Reaction Times in the Orienting and Repetition Blocks of Each Experiment as a Function of Orienting Task and Response Type

\begin{tabular}{|c|c|c|c|c|c|c|c|c|c|c|c|c|}
\hline \multirow[b]{3}{*}{ Measure } & \multicolumn{8}{|c|}{ Orienting blocks } & \multirow{2}{*}{\multicolumn{4}{|c|}{$\frac{\text { Repetition blocks }}{\text { New word pairs }}$}} \\
\hline & \multicolumn{4}{|c|}{ No-signal } & \multicolumn{4}{|c|}{ Signal-respond } & & & & \\
\hline & $\mathrm{CY}$ & $\mathrm{CN}$ & RY & RN & $\mathrm{CY}$ & $\mathrm{CN}$ & RY & RN & $\mathrm{CY}$ & $\mathrm{CN}$ & RY & RN \\
\hline \multicolumn{13}{|c|}{ Experiment 1} \\
\hline RT & 1,054 & 1,160 & 1,083 & 1,183 & 774 & 926 & 870 & 909 & 962 & 1,020 & 985 & 1,041 \\
\hline$\%$ & 93 & 93 & 94 & 94 & 77 & 79 & 83 & 79 & 93 & 96 & 94 & 94 \\
\hline \multicolumn{13}{|c|}{ Experiment 2} \\
\hline RT & 1,028 & 1,102 & 1,074 & 1,114 & 890 & 935 & 909 & 919 & 934 & 994 & 993 & 1,033 \\
\hline$\%$ & 92 & 94 & 91 & 93 & 85 & 84 & 88 & 84 & 93 & 96 & 95 & 96 \\
\hline \multicolumn{13}{|c|}{ Experiment 3} \\
\hline RT & 925 & 1,000 & 965 & 1,031 & 909 & 992 & 933 & 1,012 & 891 & 956 & 925 & 977 \\
\hline$\%$ & 92 & 93 & 94 & 92 & 90 & 92 & 93 & 90 & 93 & 95 & 93 & 93 \\
\hline \multicolumn{13}{|c|}{ Experiment 4} \\
\hline RT & 1,040 & 1,133 & 1,096 & 1,152 & 863 & 824 & 866 & 914 & 1,001 & 1,074 & 1,024 & 1,078 \\
\hline$\%$ & 91 & 92 & 92 & 92 & 86 & 79 & 81 & 83 & 92 & 95 & 92 & 93 \\
\hline \multicolumn{13}{|c|}{ Experiment 5} \\
\hline RT & 986 & 1,037 & 990 & 1,053 & 950 & 1,028 & 999 & 1,049 & 925 & 972 & 989 & 1,043 \\
\hline$\%$ & 92 & 93 & 89 & 90 & 90 & 92 & 83 & 88 & 90 & 93 & 89 & 91 \\
\hline
\end{tabular}

Note. $\mathrm{RT}=$ reaction time; $\%=$ percent correct; $\mathrm{C}=$ category; $\mathrm{R}=$ rhyme; $\mathrm{Y}=$ yes; and $\mathrm{N}=$ no.

The mean reaction times in each experiment are displayed in Table A1. The orienting block data include reaction times from no-signal trials and reaction times from those stop-signal trials on which subjects failed to inhibit their responses (i.e., signalrespond reaction times). The signal-respond reaction times are collapsed across stop-signal delay. The repetition block data include only the reaction times to new word pairs. The interested reader can calculate reaction times in the other conditions (i.e., no-signal and stop-signal conditions) by subtracting the benefit scores reported in Tables 2-6 from the reaction times to new word pairs in Table A1.

Mean reaction times for the overt response to the stop signal in Experiments 2 and 3 are presented in Table A2. Note that in both experiments, reaction time to the stop signal tends to decrease as tone delay increases, reflecting the typical psychological refractory period. However, the decrease in Experiment 2 seems less dramatic than the decrease in Experiment 3. This difference may stem from the fact that subjects inhibited about half of the re-
Table A2

Mean Reaction Times for the Overt Response to the Tone in the Orienting Blocks of Experiments 2 and 3 as a Function of Orienting Task,

Response Type, and Tone Delay

\begin{tabular}{llllll} 
& & \multicolumn{4}{c}{ Tone delay } \\
\cline { 3 - 6 } Task & Response & 200 & 500 & 800 & 1,100 \\
\hline & & \multicolumn{3}{c}{ Experiment 2} \\
Category & Yes & 557 & 504 & 493 & 457 \\
& No & 541 & 502 & 488 & 457 \\
& Yes & 544 & 523 & 487 & 462 \\
& No & 532 & 479 & 515 & 474 \\
\hline
\end{tabular}

\begin{tabular}{llllll} 
Category & Yes & 925 & 644 & 456 & 409 \\
& No & 953 & 720 & 524 & 431 \\
Rhyme & Yes & 913 & 681 & 514 & 413 \\
& No & 968 & 753 & 560 & 443 \\
\hline
\end{tabular}




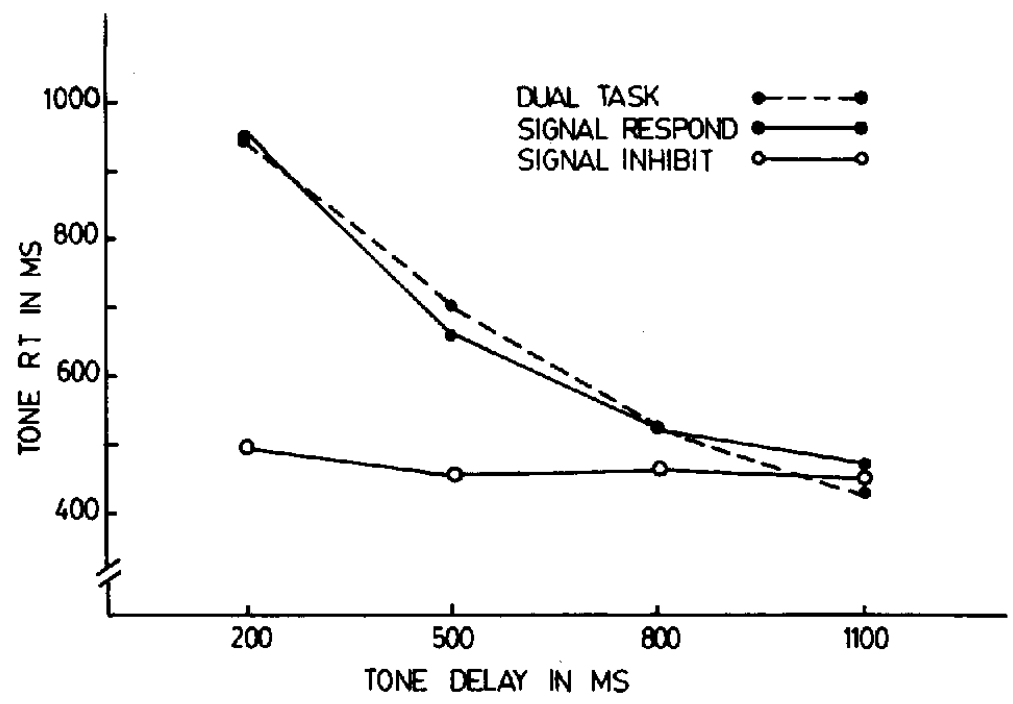

Figure A1. Reaction time to the tone as a function of stop-signal delay in Experiments 2 and 3, averaged over orienting task and response type. (Dual Task $=$ Experiment 3; Signal Respond = Experiment 2 data from trials on which a signal occurred and subjects failed to inhibit their responses; Signal Inhibit = Experiment 2 data from trials on which a signal occurred and subjects succeeded in inhibiting their responses.)

sponses in Experiment 2 versus inhibiting virtually none of the responses in Experiment 3: Inhibited responses produce less of a refractory effect than do uninhibited responses or responses that escape inhibition. This effect can be seen in Figure Al, in which the reaction times to the tone in Experiment 2 are partitioned according to whether or not subjects inhibited the concurrent response to the word pair. Responses that escaped inhibition show the same refractory effect as the uninhibited responses in Experiment 3, whereas inhibited responses show a very weak refractory effect. The weaker refractory effects following inhibited responses suggests that a major source of dual-task interference may be competition for the response system: Inhibiting the response apparently has little effect on the underlying thought, yet it virtually eliminates interference with a concurrent response. This observation is remarkable because very few manipulations eliminate dualtask interference so dramatically, but a full discussion of its implications is beyond the scope of this article. They will be addressed fully in a forthcoming paper.

Received September 9, 1983

Revision received April 8, 1985 